\title{
Ductile Shear Damage Model for Porous Materials
}

\author{
Larbi Siad $^{1}$, Sophie C. Gangloff ${ }^{1}$ and Frédéric Bohr ${ }^{2}$ \\ 1. BIOS EA4691, University of Reims, 1 rue du Maréchal Juin, Reims 51096, France \\ 2. LISM EA 4695, University of Reims, Moulin de la Housse, Reims 51687, France
}

Received: June10, 2014 / Accepted: June 20, 2014 / Published: June 25, 2014.

\begin{abstract}
Based on a pre-existing yield function, an extended version of the well-known Gurson-Tvergaad-Needleman (GTN)isotropic hardening model is presented in this investigation. The yield function of the proposed constitutive model possesses the distinctiveness to be more accurate for arbitrary void volume fraction and especially to explicitly depend upon the third stress invariant. As a numerical example, the presented constitutive model and, for the purpose of comparison, the GTN model, are used to analyse the necking of a round tensile bar and the two-dimensional simple dynamic shearing problem. The numerical results highlight similarities, good agreement as long as softening initiation of specimen is not reached, and discrepancy as soon as failure of specimen starts, between the proposed model and the GTN model.
\end{abstract}

Key words: Constitutive modelling, ductile failure, micromechanics, porous materials.

\section{Introduction}

The Gurson-Tvergaad-Needleman (GTN) model [1-4], the first micromechanical model for ductile fracture which introduces a strong coupling between deformation and damage, the material at hand is assumed to be composed of a dense matrix and spherical voids. Its yield function is affected by the presence of voids in the sense that when the void volume fraction (vvf) accumulates, the material softens and loses its capability to carry loads. The GTN's plastic potential is based on an assumption of spherical voids in an axisymmetric stress state. When the stress triaxiality (The stress triaxiality is the hydrostatic stress to equivalent stress ratio) is high enough, the voids remain near spherical and in such cases the ductile fracture process is rather well described by the GTN model. However, it is known that non-spherical voids develop at low stress triaxiality [2]. Accounting for the void shape evolution and its effect on the mechanical behavior, and a coalescence rule based upon failure of

Corresponding author: Larbi Siad, Ph.D., professor. research fields: plasticity and damage of materials and computational micromechanics. E-mail: larbi.siad@univ-reims.fr. the intervoid ligament has been considered in Refs. [5-7]. It has also been observed in Ref. [8] that void shape, growth and coalescence are strongly related to both the stress triaxiality and the degree of shear loading defined by the Lode angle which is related to the third stress invariant. In addition, if void nucleation is disregarded, the GTN model cannot describe ductile failure evolution for shear stress close to zero since, according to this constitutive model, the void growth mechanism requires hydrostatic tension within the material. However, continued softening and then ductile failure is known to occur at low triaxiality and even in situations of pure shear $[9,10]$.

Modifications of the GTN model incorporating damage growth under low triaxiality straining for shear dominated stress states have been recently proposed. In Ref. [11], the modifications retains the isotropy of the original GTN model by making use of the third stress invariant to distinguish shear dominated states. For this extension, the damage parameter is no longer the geometrically well-defined void volume fraction. It is based on the notion that the volume of voids undergoing shear may not increase, but void deformation and reorientation contribute to softening 
and constitute an effective increase in damage [12]. Alternatively, in order to phenomenologically account for the accumulative damage of voided solids due to shear deformation, the McClintock's shear failure criterion has been modified in Ref. [13] where the void shear mechanism and fracture initiation are represented through a dependence on the Lode angle.

An extension of the GTN's plastic potential $\phi_{G T}$ is proposed in Refs. [14, 15] where the authors, investigating three arrangements of spherical voids over a wide range of $\operatorname{vvf} f$, focused their study on the determination of yield surfaces for porous materials using a huge number of finite element simulations. For each of the considered three void arrangements, namely Simple Cubic (SC), Body-Centred Cubic (BCC) and Face-Centred Cubic (FCC) arrays, many thousand yield points was determined by monotonically increasing arbitrarily-prescribed macroscopic strain. The obtained yield points was fitted by a new yield function $\phi$ which turned out to be similar to $\phi_{G T}$ for $f$ ranging between a very small value to the percolation threshold found to be equal to 0.89 . Moreover, all introduced parameters may be expressed as functions of the parameters characterizing the GTN's plastic potential $\phi_{G T}$ which, in addition, is retrieved from the plastic potential $\phi$ when some of its parameters are set to be equal to appropriate values (refer to Section 2). As a novelty, the yield function $\phi_{G T}$ was found to explicitly depend upon the third stress invariant. In this investigation, a constitutive model, referred to the present model in this paper, is used to describe the progressive damage in ductile solids resulting in material softening and, ultimately, loss of its stress carrying capacity. Numerical aspects are addressed concerning the integration of the proposed constitutive rate equations. With the use of the backward Euler integration scheme, a numerical algorithm implicit in all variables as well as the corresponding algorithmic operator was developed [16]. In order to demonstrate the global accuracy and stability of the numerical solution, finite element damage simulations accounting for finite strain and using both the proposed model and, for the purpose of comparison, the GTN isotropic hardening model are performed for both the traditional ductile solid problems of necking of a round tensile bar [17] and the two-dimensional simple shearing problem.

\section{Constitutive Model Description}

The most popular model describing constitutive elastic plastic equations that account for the effect of ductile damage development is that suggested by Gurson and phenomenologically improved by Tvergaard [1-4], which makes use of an approximate yield condition

$$
\varphi_{G T}(\sigma ; H)=\left(\frac{\underline{p}}{\sigma}\right)^{2}+2 q_{1} \operatorname{csch}\left(\frac{3 q_{2} p}{2 \sigma}\right)-1-q_{1}^{2} f^{2}=0
$$

for a material containing a volume fraction $f$ of voids. In yield condition (1), the macroscopic Cauchy stress tensor $\sigma$ is resolved as

$$
\sigma=-p I+\frac{2}{3} q n \text { with } n=\frac{3}{2} \frac{\sigma^{\prime}}{q}
$$

where, $p=-1 / 3 \operatorname{tr} \sigma$ represents the hydrostatic pressure, $I$ is the second order identity tensor, $\sigma^{\prime}$ is thedeviatoric stress tensor, and $q=\left(3 / 2 \sigma^{\prime}: \sigma^{\prime}\right)^{1 / 2}$ is the von Mises stress, $\bar{\sigma}$ is the effective flow stress of the damage-free matrix material which is a function of the effective plastic strain $\bar{\varepsilon}^{p},\left(q_{1}, q_{2}\right)$ are the Tvergaard parameters, and $H=\left(H_{1}, H_{2}\right)$ is a vector comprising the scalar state variables $H_{1}=\bar{\varepsilon}^{p}$ and $H_{2}=$ $f$. An extension of the GTN's plastic potential $\phi_{G T}$ with no extra parameters which fits the numerical data well and is valid for all void volume fractions and triaxial stress states has recently been proposed in Refs. [14, 15]. The following subsection gives a useful brief review of the concerned yield function; for more detailed explanations of the subject, we refer the reader to one of the mentioned papers.

\subsection{A Pre-Existing Yield Criterion}

The porous ductile materials studied in Refs. [14, 15] contain spherical empty voids arranged in cubic arrays, 
namely, simple cubic (SC), Body-Centred Cubic (BCC) and Face-Centred Cubic (FCC) arrays. FEA was used to simulate unit cells and the macroscopic yield surfacesof the porous materials were obtained using the probing technique which goal is to obtain a yield function in an analytical expression that can be used in continuum studies. The matrix material is almost rigid, perfectly plastic and unit cells were meshed with cubes. Depending on the unit cell at hand, the void volume fractions $\mathrm{f}$ considered range from 0.02 to around 0.90 (percolation threshold of the matrix material).

As a consequence of the lattice periodicity, all outer planes of the one-eight unit cell have to behave only as rigid moveable planes in coordinate directions during the process of loading. In dyadic notation, the macroscopic strain tensor $\varepsilon$ is qualitatively given by:

$$
\varepsilon=\varepsilon_{x} e_{x} \otimes e_{x}+\varepsilon_{y} e_{y} \otimes e_{y}+\varepsilon_{z} e_{z} \otimes e_{z} \varepsilon_{y}
$$

where, for each fixed $f$, the macroscopic principal strains $\varepsilon_{x}, \varepsilon_{y}$ and $\varepsilon_{z}$ were randomly chosen. Let $I_{3}$ and $J_{3}$ be the determinant of the stress tensor $\sigma$ and the third stress invariant of the deviatoric stress tensor $\sigma^{\prime}$ respectively: $I_{3} \equiv \operatorname{det} \sigma, J_{3} \equiv 1 / 3 \operatorname{tr}{\sigma^{\prime}}^{3}$; $I_{3}$ and $J_{3}$ are related by $I_{3}=J_{3}+p q^{2} / 3-p^{3}$.

Two definitions of macroscopic yield stress has been used in Refs. [14, 15]: according to definition (1) the secant material's modulus is $95 \%$ of its original value, whereas the definition (2) states that yielding occurs as soon as the equivalent stress is at its maximum. In stress space ( $p, q, I_{3}$ ), yield points were found by monotonically increasing macroscopic strain with fixed ratios until the macroscopic equivalent stress reaches a maximum. For each of the three cubic unit cells, a least-squares fit with an extension of the GTN's yield Eq. (1) was found only approximately with deviations becoming more pronounced as pressure $p$ or void volume fraction fwere increased. To account for the observed tri-lobed numerical data pattern, the Gurson-Tvergaard-like yield criterion proposed in Refs. [14, 15] can be expressed as:

$$
\begin{aligned}
& \varphi(\sigma, H)=\left(\frac{\underline{\underline{\sigma}}}{\sigma}\right)^{2}+2 a_{1} \csc \left(\frac{3 a_{2} p}{2 \sigma}\right)-1-a_{1}^{2} \\
& +s(f) \frac{p}{\sigma}\left(\frac{I_{3}}{-3}+\frac{p^{3}}{\sigma}+\frac{1}{\sigma}\left[\frac{p}{\sigma}\left[2 a_{1} \operatorname{csch}\left(\frac{3 a_{2} p}{2 \sigma}\right)-1-a_{1}^{2}\right]\right)=0\right.
\end{aligned}
$$

Note that for the extended yield function $\phi$, the $q$-like parameters $a_{1}$ and $a_{2}$ depend on $f$ for the threeconsidered microstructures, that is $a_{1}=a_{1}(f)$, $a_{2}=a_{2}(f)$. The yield function $\phi$ is linear with respect to $I_{3}$, with coefficient proportional to the hydrostatic pressure $p$. The parameter $s$ also depending on $f$, determines the influence of the new term in the yield condition (4) which reduces to that of the GTN model (1) for $s=0, a_{1}=q_{1} f$ and $a_{2}=q_{2}$. Whenever the constantsis non-zero, there is an effect of the third stress invariant $I_{3}$ on the plastic flow.

Clearly the yield function $\phi$ contains three functions of the vvf $f$, namely $a_{1}, a_{2}$ and $s$ which are slightly different for each of the three cubic microstructures. In Refs. [14, 15], the authors stated that their finite element data were well-fit by expressing the parameters $a_{1}$ and $a_{2}$ as:

$$
a_{1}=\left(\frac{f}{f_{p}}\right)^{n}+A\left[\left(\frac{f}{f_{p}}\right)-\left(\frac{f}{f_{p}}\right)^{m}\right]
$$

where, $f_{p}$ is the percolation threshold of the microstructure and $n, m, A, Y$ and $Z$ are constant parameters. Note that (1) for $f=0$ and $a_{1}=0$, the yield Eq. (3) becomes the classical von Mises one; and (2) when $f=f_{p}$ and $a_{1}=1$, the material becomes plastic under any applied load since the matrix material is no longer connected. Regarding the parameters, if its value is too large, the tri-lobed pattern of the macroscopic yield surface almost degenerates into an equilateral triangle at some values of pressure $p$.

The yield surface becomes non-convex as $p$ is decreased further towards its maximum value given by: 


$$
p_{\max }=\left(\frac{2}{3 a_{2}}\right) \operatorname{csch}^{-1}\left(\frac{1+a_{1}^{2}}{2 a_{1}}\right)
$$

In order to only consider convex yield criteria, it was suggested in Refs. [14, 15] to put an upper-bound on the parameters depending on $p_{\max }$.

\subsection{The Proposed Rate-Independent Plasticity Model}

In non-linear solid mechanics, the material behavior is often described by a rate-form constitutive equation. In the particular case of the proposed rate-independent plasticity model, it is assumed that the macroscopic rate of strain tensor, $\dot{\varepsilon}$, is written as the sum of an elastic part $\dot{\varepsilon}^{e}$ and a plastic part $\dot{\varepsilon}^{p}$, and the stressrate $\dot{\sigma}$ depends linearly on the elastic strain rate tensor $\dot{\varepsilon}^{e}$. Subsequently, the corresponding constitutive equations can be written in a rate format as

$$
\begin{aligned}
& \dot{\varepsilon}=\dot{\varepsilon}^{e}+\dot{\varepsilon}^{p} \quad, \dot{\sigma}=C^{e}:\left(\dot{\varepsilon}-\dot{\varepsilon}^{p}\right) \\
& \dot{\varepsilon}^{p}=\dot{\lambda} r(\sigma ; H), \dot{H}=\dot{\lambda} h(\sigma ; H)
\end{aligned}
$$

where $C^{e}$ is the elastic moduli tensor which can depend on the current stress state, $r$ is the direction of the plastic flow which depends on the current stress and on a finite set of plastic internal variables $H$ accounting for history effects and $h$ is the direction of the rate of these plastic internal variables. As previously mentioned, $H$ characterizing the current state of the ductile porous material includes the void volume fraction $f$ and the effective plastic strain $\bar{\varepsilon}^{p}$ governing flow of the damage-free matrix material, that is $H=\left(H_{1}, H_{2}\right)$, with $H_{1}=\bar{\varepsilon}^{p}$ and $H_{2}=f$.

In associated plasticity, $r$ is the gradient of the yield function:

$$
\begin{aligned}
& r_{i j}(\sigma ; H)=\frac{\partial \phi}{\partial \sigma_{i j}}\left(p, q, J_{3} ; H\right) \\
& =-\frac{1}{3} \frac{\partial \phi}{\partial p} \delta_{i j}+\frac{3}{2 q} \frac{\partial \phi}{\partial q} \sigma_{i j}^{\prime}+\frac{\partial \phi}{\partial J_{3}} \sigma_{i k}^{\prime} \sigma_{i k j}^{\prime}-\frac{2}{9} \frac{\partial \phi}{\partial J_{3}} q^{2} \delta_{i j}
\end{aligned}
$$

Then the plastic strain rate $(7)_{3}$, is given by:

$$
\varepsilon^{p}=\dot{\lambda}\left(-\frac{1}{3} \frac{\partial \phi}{\partial p} I+\frac{\partial \phi}{\partial \sigma^{2}} \sigma_{i j}^{-}-\frac{2}{9} \frac{\partial \phi}{\partial J_{3}} q^{2} I\right)
$$

The plastic strain rate is trivially decomposed into volumetric $\varepsilon_{v}^{p}$ and deviatoric $\varepsilon_{q}^{p}$ parts which facilitate development of the integration algorithm:

$$
\dot{\varepsilon}^{p}=1 / 3 \dot{\varepsilon}_{v}^{p} I+\dot{\varepsilon}_{q}^{p}
$$

Using Eq. (9), $\varepsilon_{v}{ }^{p}$ and $\varepsilon_{q}{ }^{p}$ can be expressed in terms of the yield function $\phi$ as:

$$
\dot{\varepsilon}_{v}^{p}=-\dot{\lambda} \frac{\partial \phi}{\partial p} \text { and } \dot{\varepsilon}_{q}^{p}=\dot{\lambda}\left(\frac{\partial \varphi}{\partial \sigma}-\frac{2}{9} \frac{\partial \varphi}{\partial J_{3}} q^{2} I\right)
$$

It should be noted at this stage of calculation that, due to the presence of the third stress invariant $J_{3}$ in the expression of the yield function $\phi$, and in view of expression (2), the deviatoric component $\varepsilon_{q}^{p}$ cannot be put in the form $\varepsilon_{q}^{p}=\varepsilon_{q}^{p} n$, where $n$ is the deviatoric strain rate tensor normal to the yield surface $\phi=0$ and which norm is unity. This form has turned out to be very successful for applying the implicit integration scheme based on the Aravas's method [18].

The current effective stress governing flow of the damage-free matrix material $\bar{\sigma}$ which is a function of the von Mises accumulated plastic strain $\bar{\varepsilon}^{p}$, through the hardening law:

$$
\bar{\sigma}=\bar{\sigma}\left(\bar{\varepsilon}^{p}\right)
$$

Equating the plastic dissipation rate of a representative volume element in tensor form with the plastic dissipation rate of average matrix properties results in:

$$
(1-f) \bar{\sigma} \dot{\bar{\varepsilon}}^{p}=\sigma: \dot{\varepsilon}^{p}=\dot{\lambda} \sigma: r(\sigma ; H)
$$

where $\dot{\bar{\varepsilon}}^{p}$ is an average effective plastic strain rate in the matrix material.

Two terms are present in the evolution law of the void volume fraction [2-4]: $\dot{f}=\dot{f}_{g}+\dot{f}_{n}$. Accumulation of plastic deformation around already nucleated voids causes their enlargement and subsequently an increase of the void volume fraction which, by mass conservation, writes:

$$
\dot{f}_{g}=(1-f) \dot{\varepsilon}_{v}^{p}
$$

The term $f_{g}$ strongly depends on the stress triaxiality $\mathcal{T}=-p / q$. In particular, the elastic volume variation does not induce a porosity change. The void fraction can also increase due to void nucleation by cracking of second phase particles or debonding of the matrix material from particles. The nucleated voids will grow subsequent to nucleation, but there is no plastic 
volume change associated with these nucleation events. Here, for simplicity, it is assumed that growth of newly nucleated voids is negligible in the time step in which the nucleation event occurred. Newly nucleated voids affect the yield function, Eq. (4), but not the void growth relations for that time step. Since nucleation of new voids from second phase particles is assumed to not impact void growth in the same time increment, the nucleation models can be decoupled from void growth. Strain controlled nucleation was used in the constitutive model and the nucleated void volume fraction is given by:

$$
\dot{f}_{n}=A\left(\bar{\varepsilon}^{p}\right) \dot{\bar{\varepsilon}}^{p}
$$

In this study, the normal distribution law suggested in Ref. [2] is chosen for the factor A:

$$
\mathrm{A}\left(\bar{\varepsilon}^{p}\right)=\frac{f_{N}}{s_{N} \sqrt{\pi}} \exp \left[-\frac{1}{2}\left(\frac{\bar{\varepsilon}^{p}-\varepsilon_{N}}{s_{N}}\right)^{2}\right]
$$

when $\dot{\bar{\varepsilon}}^{p}>0$. For this strain nucleation model, $f_{N}$ is the volume fraction of voids to be nucleated, $\epsilon_{N}$ is the mean value about which nucleation occurs, and $s_{N}$ is the standard deviation. Under plastic loading, void nucleation occurs as soon as the current matrix effective plastic strain, $\bar{\varepsilon}^{p}$, equals or exceeds its previous maximum. Using equations (13)-(16), the evolution of the plastic internal variables $H$, (Eq. (7) reads:

$$
\begin{aligned}
& h_{1}=\frac{\sigma: \dot{\varepsilon}^{p}}{(1-f) \bar{\sigma}}=\frac{-p \dot{\varepsilon}_{v}^{p}+\sigma^{\prime}: \dot{\varepsilon}_{q}^{p}}{(1-f) \bar{\sigma}} \\
& h_{2}=-(1-f) \frac{\partial \phi}{\partial p}+A\left(\bar{\varepsilon}^{p}\right) \dot{\bar{\varepsilon}}^{p}
\end{aligned}
$$

where the symbol: means doubly contracted tensor product.

To determine the plastic multiplier, the loading unloading conditions should be imposed in a Kuhn-Tucker form as:

$$
\dot{\lambda} \geq 0, \phi\left(p, q, J_{3}: H\right) \leq 0, \dot{\lambda} \phi\left(p, q, J_{3}: H\right)=0(18)
$$

implying that during plastic loading, $\phi=0, \lambda \geq 0$ and $\phi$ $=0$. This condition, the consistency condition, allows the determination of the plastic multiplier which specifies the magnitude of the plastic strain rate [19]. Finally, the void fraction modification $f^{*}$ introduced in Ref. [1] was intended to simulate the rapid loss of strength accompanying void coalescence.

\subsection{Numerical Integration of the Present Constitutive Model}

The calculated porous plastic material response is strongly dependent on the computational procedure for stress calculation, usually called the stress integration. The presence of the third stress invariant $J_{3}$ in the yield function $\phi$ typically results in a high degree of non-linearity and complex numerical algorithms. Implicit solution strategies for models depending on the third stress invariant have been developed, for associative isotropic elastoplastic and viscoplastic models [20], for associative elastoplastic models with kinematic hardening [21, 22] and for models of general isotropic elastoplastic geomaterials [21, 23, 24]. Generally speaking, regarding elastoplastic material, the incremental-iterative analysis consists in dividing up the applied load into a number of small increments, and within each increment, iterations are performed. A consistent algorithm has to be devised for a small increment of loading, such that the following update can be carried out:

$$
\left(\sigma_{t}, \phi_{t}, \lambda_{t}, \varepsilon_{t}, \Delta \varepsilon_{t+\Delta t}\right) \rightarrow\left(\sigma_{t+\Delta t}, \phi_{t+\Delta t}, \lambda_{t+\Delta t}, \varepsilon_{t}, \varepsilon_{t+\Delta t}\right)
$$

where the subscripts $t$ and $t+\Delta t$ refer to the beginning and the end of the current loading increment, respectively, and the update Eq. (19) is fully driven by the strain increment $\Delta \varepsilon_{t+\Delta t}$. For further details dealing with the description of various integration methods, the reader is referred to books by, for example, Simo and Hughes [19] and Anandarajah [25] where more elaborate coverage of the Closest Point Projection Method may be found.

The proposed constitutive Eqs. (4), (9), (14-17), and the coalescence criterion based on the effective porosity $f^{*}$ introduced in Ref. [17] are integrated using 
a fully implicit scheme which allows the calculation of the consistent tangent operator [19, 25, 16]. The final stress and hardening parameters are determined solving the non-linear equations iteratively so that the stress increment fullfills the consistency condition. In each time increment $[t, t+\Delta t]$, the total strain increment $\Delta \varepsilon_{t+\Delta t}=\varepsilon_{t+\Delta t}-\varepsilon_{t}$ is assumed to be known and generally constant over it. The aim of the proposed integration scheme is to compute the evolution of the whole state variables over $[t, t+\Delta t]$, and in particular their values at $t+\Delta t$. These state variables must satisfy the constitutive Eqs. (4), (9), (14), (15), (17), the coalescence criterion, along with the initial conditions at time $t$. In the Closest Point Projection Method the equilibrium equations are written at the end of the increment and the stress increment corresponding to any given strain increment must be computed iteratively.

$\Delta \varepsilon_{t+\Delta t}=\Delta \varepsilon_{t+\Delta t}^{e}+\Delta \varepsilon_{t+\Delta t}^{p} \quad$ is the adequate additive decomposition as long as the elastic strains are small. The elastic predictor is used to check the loading unloading event. The closest point projection method is an elastic predictor-plastic corrector method that seeks to satisfy consistency at the end of the increment. The objective is to find $\Delta \sigma_{t+\Delta t}$ and $H_{t+\Delta t}$ bythe following backward Euler (implicit) equations:

$$
\begin{aligned}
& \sigma_{t+\Delta t}=\sigma_{t}+\Delta \sigma_{t+\Delta t}=\sigma_{t+\Delta t}^{T}-C^{e}: \Delta \varepsilon_{t+\Delta t}^{p} \\
& =\sigma_{t+\Delta t}^{T}-\Delta \lambda_{t+\Delta t} C^{e}: r_{t+\Delta t}
\end{aligned}
$$

where, $\sigma_{t+\Delta t}^{T}=\sigma_{t}+C^{e}: \Delta \varepsilon_{t+\Delta t}$ is the trial elastic stress. The elastic moduli tensor $C^{e}$ is assumed to be constant and to be determined by elastic bulk modulus $K$ and the elastic shear modulus $G$. Its components are then given by:

$$
C_{i j k l}^{e}=\left(K-\frac{2}{3} G\right) \delta_{i j} \delta_{k l}+2 G \delta_{i k} \delta_{i j l}
$$

where, $\delta_{i j}$ is the Kronecker symbol and the second order tensor $C^{\mathrm{e}}$ : $r$ is immediately split up into spherical and deviatoric parts:

$$
C^{e}: r=K r_{v} I+2 G r^{\prime}
$$

Hence, Eq. $(20)_{2}$ may be written as:

$$
\sigma_{t+\Delta t}=\sigma_{t+\Delta t}^{T}-K \Delta \varepsilon_{v t+\Delta t}^{p} I-2 G \Delta \varepsilon_{q t+\Delta t}^{p}
$$

The evolution of the plastic internal variables $H$ can be written as functions of stresses, plastic strain increments, and plastic internal variable themselves:

$$
H_{t+\Delta t}=H_{t}+\Delta \lambda_{t+\Delta t} h\left(\Delta \varepsilon_{t+\Delta t}^{p}, p_{t+\Delta t}, q_{t+\Delta t}, J_{3 t+\Delta t} ; H_{t+\Delta t}\right)
$$

The unknowns $P_{t+\Delta t}, \sigma_{t}^{\prime}+\Delta t$ and $H_{t}+\Delta t$ to be determined are such that:

$$
\phi\left(p_{t+\Delta t}, q_{t+\Delta t}, J_{3 t+\Delta t} ; H_{t+\Delta t}\right)=0
$$

By combining appropriate derived relations from the above equations in order to eliminate $H$ and $\lambda$, the relation $\sigma=C^{e p}: \varepsilon$, where $C^{e p}$ is the consistent tangent operator, can be obtained. The corresponding algorithm is programmed in two user subroutines, Umat for implicit analyses and Vumat for explicit ones, which are called by Abaqus/Standard and Abaqus/Explicit [26], respectively, at each element integration point, for each increment, and during each load step.

\section{Examples Computations}

\subsection{Rounded Smooth Bar Tensile Test}

This section illustrates the use of the present model through the analysis of damage and failure of smooth bar subject to axial tensile test. The traditional necking analysis of a round bar has been investigated. Uniaxial tensile test of a smooth bar is often used to study the ductile void growth in metals for which the formation of a neck triggers the material instability. A detailed finite element analysis of the formation of the crack at the center of the neck and its subsequent growth leading to cup-cone fracture can be found, for instance, in Ref. [5]. The formation of the neck results in a triaxial state of stress at the center of the specimen. Due to high hydrostatic tension, the growth of nucleated voids are accelerated and the material instability proceeds with the initiation of fracture at the center of the neck with linkage of adjacent voids. Numerical simulations of the behavior of a rounded smooth bar subject to tensile test have been performed. 
The rounded bar has an initial length of $2 L_{z o}$ and a radius of $L_{\rho o}$ with $L_{z o}=4 L_{\rho o} . L_{\rho o}$ is assumed to be equal to one unit. Due to symmetry about $\rho=0$ and $Z=0$, only one quarter of the specimen needs to be analyzed. A conical imperfection is introduced near the center of the specimen such that $L_{\rho o}^{Z=0}=0998 L_{\rho o}$. The geometry of the studied specimen and the original used mesh are shown in Fig. 1a.

The geometry is axisymmetric, and the deformation is assumed to be axisymmetric. Reduced integration elements (CAX4R within Abaqus) were used for efficient computations. A refined mesh is generated near the center of the rounded bar where softening and intense deformation are expected, whereas a relatively coarse mesh is used in the rest of the specimen where a rather uniform deformation is expected. The kinematic boundary conditions are symmetry about $\rho$ $=0$, symmetry about $z=0$, and all the nodes on the edge $\mathrm{z}=L_{z o}$ of the specimen are given a prescribed velocity depending on time, $v_{z}=v_{z}(t)$, in the $\mathrm{z}$ direction. (Fig. 1b). The velocity $v_{z}$ increases rapidly from zero at the start of the analysis to its maximum $30 \mathrm{~ms}^{-1}$ in $0.0025 \mathrm{~s}$ and drops back linearly to zero in another $0.0025 \mathrm{~s}$. It then remains at zero for the remainder of the analysis, (Fig. 1b).

The specimen is made of a steel alloy which work-hardening characteristics are similar to those of a power law hardening material with Young's modulus $E$, Poisson's coefficient $v$, and initial yield stress $\sigma_{o}$. Using the GTN model to simulate ductile failure, a proper choice of its eight micro-mechanistic parameters $\epsilon_{N}, s_{N}, f_{N}, f_{o}, f_{c}, f_{f}, q_{1}$ and $q_{2}$ proves tobe crucial. The damage parameters $\epsilon_{N}, s_{N}$ and $f_{N}$ related to voidnucleation, as well as the Tvergaardconstants $q_{1}$ and $q_{2}$ were fixed to typical values suggested in Ref. [1]. Before loading the material is assumed to be fully dense, that is $f_{o}=0$. Table 1 lists the material parameters used in the numerical simulations.

It is well known that the material deforms homogeneously in the axial direction before the load becomes maximum, and that the material deforms heterogeneously in the axial direction, that is necking occurs after the load becomes maximum, and then the specimen softens due coalescence of voids and eventually fracture across the neck region. Fig. 2 displays the deformed shape of the tensile bar showing necking occurs at the mid-section of the specimen.

The global response of the specimen may be specified by the average states of strain and stress within it. The nominal elongation $E_{z}^{n}=\Delta L_{z} / L_{\rho 0}$ and the relative diameter $E_{\rho}^{n}=\Delta L_{\rho} / L_{\rho}$ are chosen to assess the average strains of the specimen; $\Delta L_{z}=L_{z}-L_{z 0}$ is the axial displacement of the top of the specimen and $\Delta L_{\rho o}=L_{z}-L_{\rho}$ is the reduction of radius of its middle section. $E_{\rho}^{n}$ is used to assess the average strain in sections perpendicular to the tensile direction. The overall nominal stress is given by $\Sigma_{z}^{n}=F / \pi L_{\rho o}^{2}$, where $F$ is the resulting overall tensile load carried by the bar and determined from nodal forces. The results of the analysis are illustrated in Fig. 3. The evolution of $\Sigma_{z}^{n}$ as functions of $E_{z}^{n}$ and $E_{\rho}^{n}$ are shown in Fig. 3a and 3b, respectively, for both the present model and the GTN model. First of all, these figures clearly show that up to failure initiation points which are indicated in Fig. 3 using the $\otimes$ symbols, the agreements between predictions of considered models are quite very good. In particular, maximum loads for the present model,

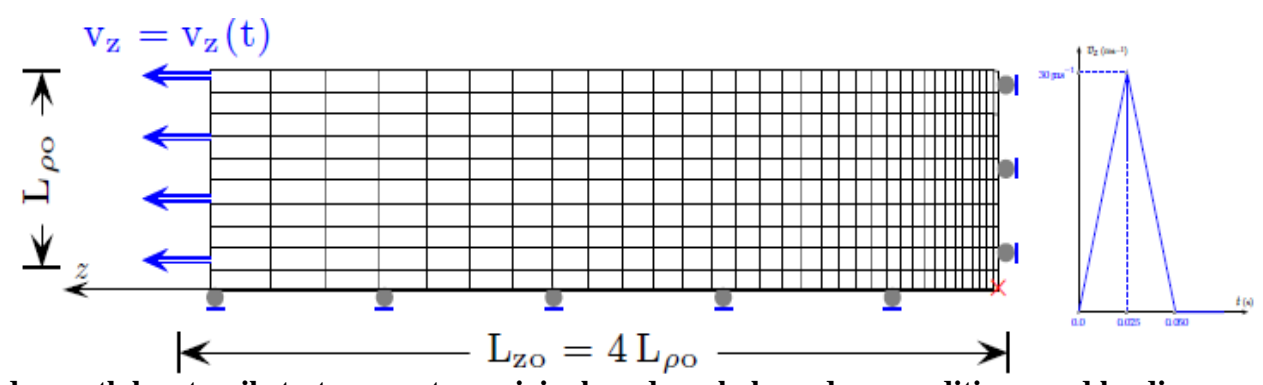

Fig. 1 Rounded smooth bar tensile test: geometry, original used mesh, boundary conditions, and loading. 
Table 1 Used materials parameters.

\begin{tabular}{lr}
\hline Elasticity & $\frac{\sigma_{o}}{E}=\frac{1}{300}, v=0.3$ \\
\hline Hardening curve & $\bar{\sigma}\left(\bar{\varepsilon}^{p}\right)=\sigma_{o}\left(1+0.033 \bar{\varepsilon}^{p}\right)^{0.1}$ \\
\hline \multirow{2}{*}{ Damage } & $q_{1}=1.5, q_{2}=1.0$ \\
& $\epsilon_{N}=0.3, s_{N}=0.1, f_{N}=0.04$ \\
& $f_{o}=0.0, f_{c}=0.15, f_{f}=0.25$ \\
\hline
\end{tabular}
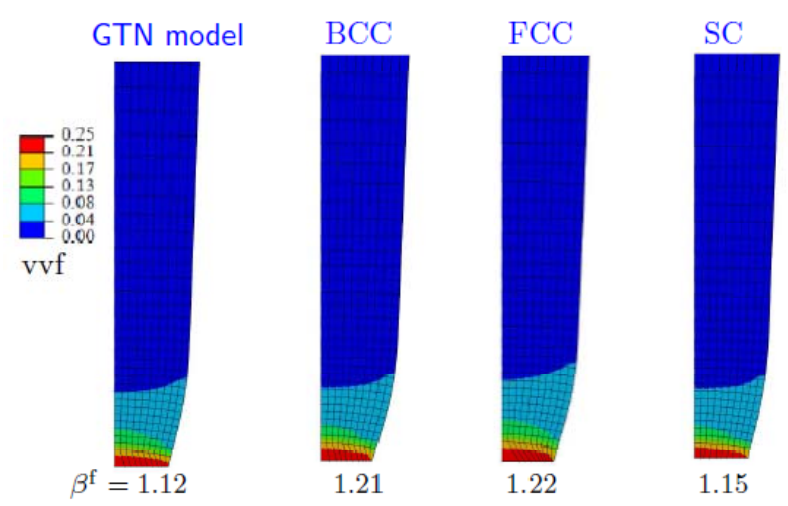

Fig. 2 Simulation of tensile tests on smooth round bar.Comparison of damage contour plots predicted by the present models and the GTN model at the final edge displacements of the specimen; $\beta^{f}=u^{z} / L_{\varrho o}$.

regardless the considered microstructure, coincide with the maximum load provided by the GTN model which is reached at strains $E_{z}^{n}$ of about $11 \%$ and $E_{\rho}^{n}$ of about $5.5 \%$.

It is worth noting that the practically coincidence of the numerical predictions also holds in the softening regime ranging from maximum load and the immediate vicinity of failure points. This fact is substantiated by the deformed configuration and the contours of the void volume fraction depicted in Fig. 2 at the final edge displacements of the specimen which are very similar. As expected, the largest amount of softening localizes around the center of the mid-section of the specimen. It is implied, therefore, that failure initiation should be expected in that area. However, the onset of failure is reached at displacements of $1.12 L_{\rho o}$ for the GTN model and of $1.21 L_{\rho o}$ for the present model with BCC microstructure, Fig. 2. The maximum loads along with the corresponding nominal strains predicted by both the present models and the GTN model are listed in Table 2.
Deviations between predictions of the present models and the GTN model are observed as soon as failure of the considered specimen starts, i.e. when the sudden specimen capacity loss occurs (Fig. 3). Thenumerical results seem to suggest that the present model based on SC microstructure is the closer one to the GTN model. The true fracture strain is the most physical experimental indicator for quantifying the resistance to damage and fracture of a material subjected to tensile loading conditions. An average true fracture strain measure $\varepsilon_{f}$ using the diameter reduction is usually used to quantify the ductility $[9,10]$. It is defined as $\varepsilon_{f}=2 \log \left(L_{\varrho o} / L_{\varrho}\right)$. It has been well known that the dependence of $\varepsilon_{f}$ on the stress triaxiality $\mathcal{T}$ can be approximated using an exponential function:
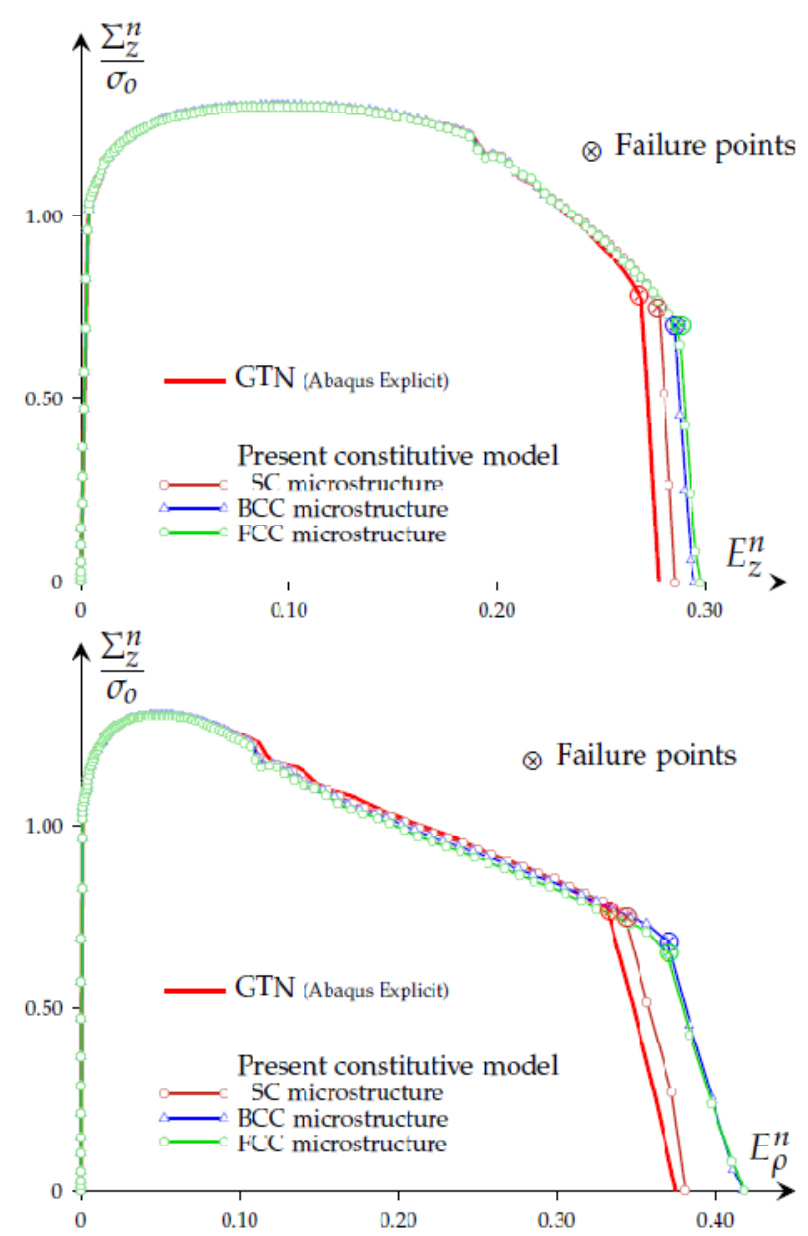

Fig. 3 Simulation of tensile tests on smooth round bar. Predictions of overall nominal stress versus overall nominal strains. 
Table 2 Maximum loads and corresponding nominal strains.

\begin{tabular}{lllll}
\hline Strain-Maximum stress & $E_{z}^{n}(\%)$ & $\left(\frac{\sum_{z}^{n} \max }{\sigma_{o}}\right)$ & $E_{\varrho}^{n}(\%)$ & $\left(\frac{\sum_{\varrho}^{n} \max }{\sigma_{o}}\right)$ \\
\hline GTN & 8.67 & 1.301 & 4.87 & 1.302 \\
Present model SC & 10.27 & 1.302 & 4.87 & 1.303 \\
Present model BCC & 9.72 & 1.303 & 5.02 & 1.303 \\
Present model FCC & 9.45 & 1.301 & 4.86 & 1.301 \\
\hline \multicolumn{2}{l}{ Table 3 Comparison of predicted failure strains. } & & & FCC \\
\hline Ductility & GTN & SC & BCC & 30.5 \\
\hline$\left(E_{z}^{n}\right)^{f}(\%)$ & 27.5 & 28.3 & 30.2 & 42.5 \\
$\left(E_{\varrho}^{n}\right)^{f}(\%)$ & 36.2 & 37.2 & 42.4 & \\
\hline
\end{tabular}

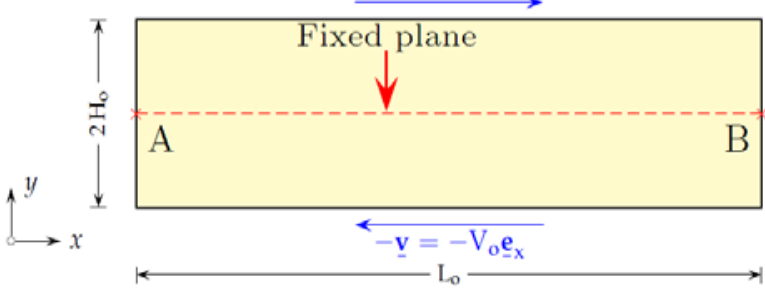

Fig. 4 Schematic of a two-dimensional simple shear problem. The layer of height $2 H_{o}$ is deformed in shear by imposed velocities $v= \pm V_{o} e_{x}$ at its upper $\left(y=H_{o}\right)$ and lower faces $\left(\mathrm{y}=-\mathrm{H}_{o}\right)$, respectively.

$\varepsilon_{f}=\alpha \exp (-\alpha \mathcal{T})$, where $\alpha$ is a material constant. The obtained failure strains are listed in Table 3. Specimen made up of GTN material has a little lower ductility than the specimen made up of present models material, regardless the considered microstructure (BCC, FCC or SC).

\subsection{Two-Dimensional Simple Dynamic Shearing Problem}

Consider a simple shearing deformation of a homogeneous layer in the $(x, y)$ plane as shown in Fig. 4. The layer has a height $2 H_{o}$ in the $y$-direction, a length $L_{o}$ in the $x$-direction, while it extends indefinitely along the z-direction. The problem at hand is two dimensional in the sense that all the quantities are only functions of space coordinates $(\mathrm{x}, \mathrm{y})$ and timet. The considered boundary conditions consist of velocity controlled boundaries. The upper and lower faces of the layer are subject to constant velocities $v=+V_{o} e_{x}$ and $v=-V_{o} e_{x}$, respectively, while the mid-face $\mathrm{AB}$ is maintained fixed. This is mathematically expressed as follows:

$$
\begin{aligned}
& v\left(x, H_{0}, t\right)=V_{0} e_{x}, v\left(x,-H_{0}, t\right)=-V_{0} e_{x}, \\
& v\left(x, 0_{0}, t\right)=0,0 \leq t \leq \infty
\end{aligned}
$$

The nominal shear $\operatorname{strain} \Gamma_{\mathrm{n}}$ is defined as the mean displacement $U_{o}$ divided by the half-thickness of the layer, $\Gamma_{n}=U_{o} / H_{o}$. The matrix material of the specimen is made of a typical hard steel which true stress-true strain curve in uniaxial tension is taken to follow the power law:

$$
\bar{\sigma}\left(\bar{\varepsilon}^{p}\right)=\sigma_{o}\left(1+F \bar{\varepsilon}^{p .26}\right)
$$

with initial yield stress $\sigma_{o}$ and a power hardening exponent equal to 0.26 . In the results presented below, the analyses were carried out for the assigned following values of various material and geometric parameters: $H_{o}=0.325 \mathrm{~mm}, L_{o}=5.08 \mathrm{~mm}, V_{o}=50$ $\mathrm{m} / \mathrm{s}, \varrho_{o}=7,830 \mathrm{~kg} / \mathrm{m}^{3}, \sigma_{0}=1,805.3 \mathrm{MPa}$ and $\mathrm{F}=$ 0.639 . The average applied strain rate is $\Gamma_{\mathrm{n}}=1.54 \times$ $10^{4} \mathrm{~s}^{-1}$.

The used damage parameters are the same as those kept for the first numerical example and are listed in Table 1. Abaqus Explicit [26] is employed to run the numerical analysis. To reduce the computer run times, specimens are modeled using four nodes plane strain reduced integration elements, CPE4R. The used mesh with element size around the midsection of the layer of approximately $16 \mu \mathrm{m}$ is shown in Fig. 6 .

The geometry of the considered shear layer and its loading are approximately similar to the one used in Ref. [9] where the authors focused their studies on the shear failure of a VAR 4340 steel sample subjected to 


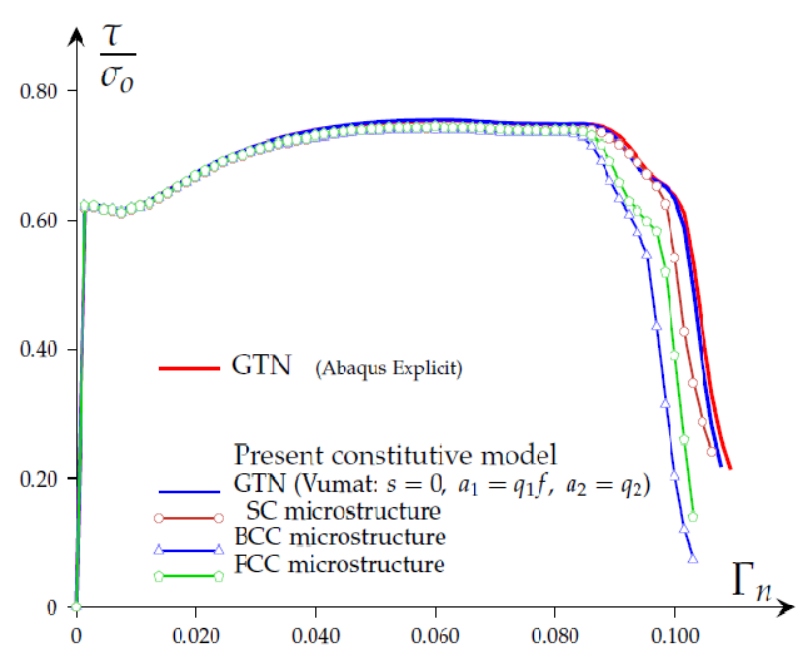

Fig. 5 Normalized nominal shear stress-nominal shear strain diagrams.

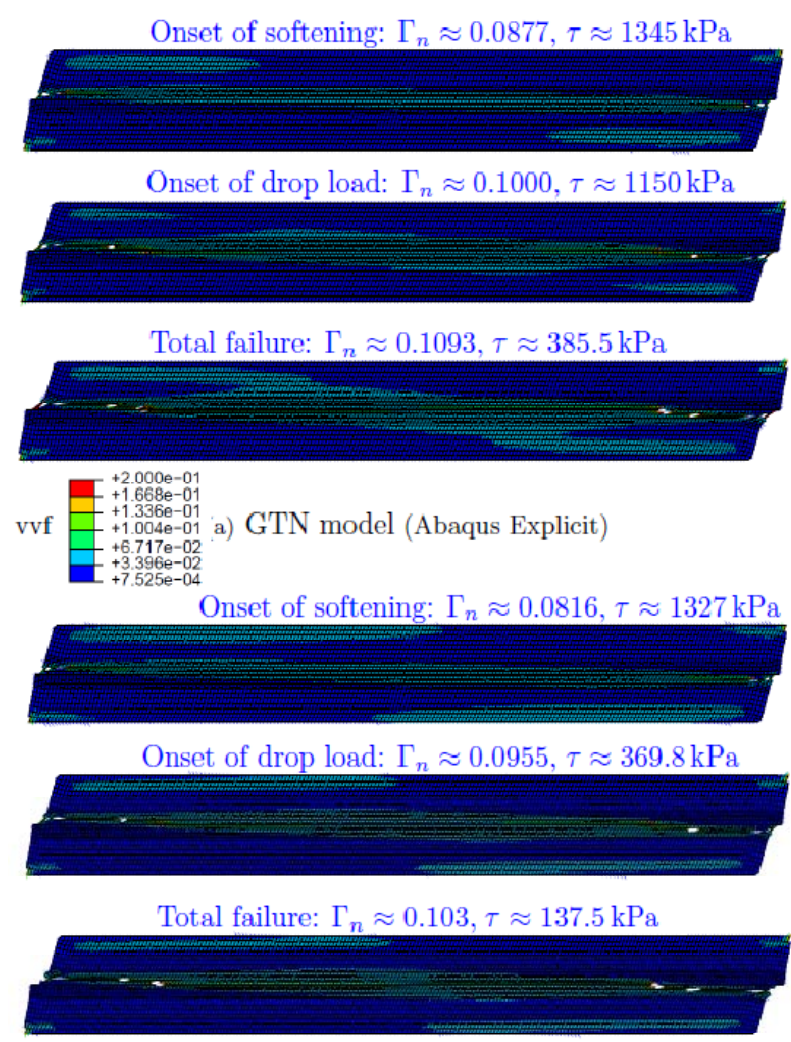

(b) Present model (BCC microstructure)

Fig. 6 Void volume fraction distribution within the considered finite porous layer at various times as shear localization forms. Comparison of damage contour plots predicted by the present BCC constitutive damage models and the GTN model from the onset of softening at the final faces displacements of the specimen.

a ballistic impact. On the basis of their experimental results, these authors observed, although rate effects play an important role in determining the material strength, a similar shear instability strain under quasi-static testing conditions.

Accordingly, the noticed softening cannot be explained in terms of (dynamic) adiabatic shear bands. In addition, the taken micrographs showed evidence of elongated micro-voids in the shear plane for fracture surfaces resulting from low triaxiality ductile failure. It was also observed in Ref. [9] that the shear instability could be delayed by increasing the interfacial strength of the secondary particle-matrix interface or by applying a compressive hydrostatic stress. Fig. 5 depicts the normalized nominal shear stress, $\tau$ at $y=H$, defined as $\tau / \sigma_{o}$, versus the nominal shear strain $\Gamma_{n}$. The materials at hand begin to strain and then exhibit uniform deformation up to the shear instability corresponding to the condition $d \tau / d \Gamma_{n}=0$, after which a shear band like begins to form. Regarding the comparison of the predictions of the used constitutive models, at first glance similar conclusions as for the smooth bar can be drawn. The numerical results based on the present model with assigned values $s=0, a_{1}=q_{1}$ and $a_{2}=q_{2}$ in the yield condition (4), in order to recover the GTN model, agree very well with the prediction of the GTN model of Abaqus Explicit [26]. Deviations between predictions of the present model and the GTN model are observed as soon as softening comes forward and become pronounced when failure of the considered specimen starts. Again, the constitutive model with SC microstructure also turns out to the closer one to the GTN model.

By way of illustration, Fig. 6 shows the contours of the void volume fraction obtained with use of the GTN model of Abaqus Explicit [26] and, for the sake of brevity, the present model based on the BCC microstructure. The chosen particular times are the onset of softening, the incipient of the drop load and the final face displacements of the specimen corresponding to its total failure. 


\section{Conclusions}

The main objective of this paper has been to address an extended version of the GTN model based on a pre-existing yield function for porous plastic materials proposed in Refs. [14, 15] and its implementation within a finite element code by writing a user material subroutine. A fully implicit stress integration scheme is used to integrate the present model. The final stresses and hardening parameters are determined solving the non-linear equations iteratively so that the stress increment satisfies the consistency condition. As first numerical example, the present model is used to perform axisymmetric simulations of the ductile necking failure of a smooth round bar and the shear failure process of a finite layer. Similar values for the damage parameters (nucleation and coalescence) have been used for both the present model and the GTN model in order to compare their ability to predict void growth to coalescence and the corresponding failure mechanism. The obtained numerical results are summarized as follows.

The global accuracy and stability of the integration procedure is illustrated from the performed simulation of the considered tensile tests. The present model has shown the potential to accurately predict all the major features of ductile failure of solids (stress triaxiality dependence).

Up to the failure initiation of the specimen, the predictions based on the finite element analysis incorporating the present model are in a close agreement with those provided by the GTN model, confirming the potential of the former constitutive model to fullfill to the requirement of transferability between different loading conditions.

Noticeable disagreement has been observed between predictions of both models at and beyond failure points of specimen. The necking round bar problem has shown that during the sudden specimen capacity loss, the present model based on SC microstructure is the closer one to the GTN model.

\section{References}

[1] A.L. Gurson, Continuum theory of ductile rupture by void nucleation and growth: Part I-yield criteria and flow rules for porous ductile media, J. Engng. Mater. Technol. 99 (1977) 2-15.

[2] V. Tvergaard, Materials Failure by Void Growth to Coalescence, Advances in Applied Mechanics, Academic Press, New York, 1994, Vol. 27, pp. 83-151.

[3] A.A. Benzerga, J.B. Leblond, Ductile Fracture by Void Growth to Coalescence, Advances in Applied Mechanics, Academic Press, New York, 2010, Vol. 44, pp. 169-305.

[4] J. Besson, Continuum models of ductile fracture: A review, Int. J. Damage Mechanics 19 (2010) 3-52.

[5] M. Gologanu, J.B. Leblond, J. Devaux, Recent extensions of Gurson's model for porous ductile metals, CISM Lectures Series, Springer-Verlag, Berlin, 1997, pp. 61-106.

[6] T. Pardoen, J.W. Hutchinson, An extended model for void growth and coalescence, J. Mech. Phys. Solids 48 (2000) 2467-2512.

[7] A.A. Benzerga, Micromechanics of coalescence in ductile fracture, J. Mech. Phys. Solids 50 (2002) 1331-1362.

[8] X. Gao, J. Kim, Modeling of ductile fracture: Significance of void coalescence, International Journal of Solids and Structures 43 (2006) 6277-6293.

[9] J.G. Cowie, M. Azrin, G.B. Olson, Microvoid formation during shear deformation of ultrahigh strength steels, Metallurgical Transactions A20A(1989)143-153.

[10] I. Barsoum, J. Faleskog, Rupture in combined tension and shear: Experiments, International Journal of Solids and Structures 44 (2007) 1768-1786.

[11] K. Nahshon, J.W. Hutchinson, Modification of the Gurson model for shear failure, European Journal of Mechanics A Solids 27 (2008) 1-17.

[12] Tvergaard, K.L. Nielsen, Relations between a micro-mechanical model and a damage model for ductile failure in shear, J. Mech. Phys. Solids 58 (2010) 1243-1252.

[13] L. Xue, Constitutive modeling of void shearing effect in ductile fracture of porous materials, Engineering Fracture Mechanics 75 (2008) 3343-3366.

[14] D.L. Sean McElwain, A.P. Roberts, A.H. Wilkins, Yield criterion for porous materials subjected to complex stress states, Acta Materialia 54 (2006) 1995-2002.

[15] D.L. Sean McElwain, A.P. Roberts, A.H. Wilkins, Yield functions for porous materials with cubic symmetry using different definitions of yield, Advanced Engineering Materials 8 (2006) 870-876.

[16] L. Siad, Ductile damage analysis based on a J3-GTN-like model, Journal of Multiscale Modelling 3 (2011) 1-27. 
[17] V. Tvergaard, A. Needleman, Analysis of the cup-cone fracture in a round tensile bar, Acta Metallurgica 32 (1984) 157-169.

[18] N. Aravas, On the numerical integration of a class of pressure-dependent plasticity models, Int. J. Numer. Meth. Engng. 24 (1987) 1395-1416.

[19] J. Simo, J.R. Hughes, Computational Inelasticity, Springer-Verlag, New York, 1998.

[20] J.L. Chaboche, G. Cailletaud, Integration methods for complex plastic constitutive equations, Comput. Methods Appl. Mech. Engrg. 133 (1996) 125-155.

[21] R.I. Borja, K.M. Sama, P.F. Sanz, On the numerical integration of three-invariant elastoplastic constitutive models, Comput. Methods Appl. Mech. Engrg. 192 (2003) 1227-1258.

[22] C.D. Foster, R.A. Regueiro, A.F. Fos sum, R.I. Borja, Implicit numerical integration of a three-invariant, isotropic/kinematic hardening cap plasticity model for geomaterials, Comput. Methods Appl. Mech. Engrg. 194 (2005) 5109-5138.

[23] X. Wang, L.B. Wang, L.M. Xu, Formulation of the return mapping algorithm for elastoplastic soil models, Computers and Geotechnics 31 (2004) 315-338.

[24] C. Tamagnini, R. Castellanza, R. Nova,A generalized backward Euler algorithm for the numerical integration of an isotropic hardening elastoplastic model for mechanical and chemical degradation of bonded geomaterials, Int. J. Numer. Anal. Meth. Geomech. 26 (2002) 963-1004.

[25] A. Anandarajah, Computational Methods in Elasticity and Plasticity, Solids and Porous Media, Springer-Verlag, New York, 2010.

[26] Abaqus Explicit, Version 6.4. HKS, Inc., Providence, RI, 2004.

[27] J. Besson, Axisymmetric notched bar, mechanical tests and constitutive laws (in french), Hermès Science, Paris, 2001, pp. 319-351.

[28] N. Bonora, Identification and measurements of ductile damage parameters, The Journal of Strain Analysis for Engineering Design 34 (1999) 463-478.

[29] F.A. McClintock, A criterion for ductile fracture by the growth of holes, Journal of Applied Mechanics 33 (1968) 363-371.

[30] J.R. Rice, D.M. Tracey, On the ductile enlargement of voids in triaxial stress fields, J. Mech. Phys. Solids 17 (1969) 201-217.

[31] J.W. Hancock, A.C. Mackenzie, On the mechanism of ductile fracture in high-strength steels subjected to multi-axial stress-states, J. Mech. Phys. Solids 24 (1976) 147-169. 